\title{
Sectionectomy Is Suitable for Patients with T2 Hepatocellular Carcinoma according to the Modified International Union against Cancer TNM Classification
}

\author{
Shun-Ichi Ariizumi Satoshi Katagiri Hideo Katsuragawa Yoshihito Kotera \\ Masakazu Yamamoto \\ Department of Surgery, Institute of Gastroenterology, Tokyo Women's Medical University, Tokyo, Japan
}

\begin{abstract}
Key Words
Hepatocellular carcinoma - Anatomical resection •

Sectionectomy $\cdot$ TNM classification
\end{abstract}

\begin{abstract}
Background/Aims: The effectiveness of systematized hepatectomy in the modified International Union Against Cancer (UICC) staging classification for hepatocellular carcinoma (HCC) has not been clarified in detail. Methods: We retrospectively studied 406 patients with UICC T1 HCC and 124 patients with $\mathrm{T} 2 \mathrm{HCC}$ who underwent initial curative hepatectomy from 1994 through 2003. Outcomes after sectionectomy or larger resection and segmentectomy or smaller resection for patients with $\mathrm{T} 1 \mathrm{HCC}$ and $\mathrm{T} 2 \mathrm{HCC}$ were examined. Results: The overall recurrence-free survival rate and survival rate of sectionectomy or larger resection at 5 years (45 and 79\%, respectively) did not significantly differ from those of segmentectomy or smaller resection (38 and 81\%, respectively) in patients with $\mathrm{T} 1 \mathrm{HCC}$. However, in patients with $\mathrm{T} 2 \mathrm{HCC}$, the 5 -year recurrence-free survival rate and survival rate after sectionectomy or larger resection (37 and $71 \%$, respectively) were significantly different from those after segmentectomy or smaller resection $(6 \%: p<0.0001$, and $35 \%: p=0.0027$, respectively). Multivariate analysis showed
\end{abstract}

sectionectomy or larger resection to be a significant independent prognostic factor for recurrence-free survival and survival in patients with T2 HCC. Conclusions: Sectionectomy or larger resection prevents intrahepatic recurrence of $\mathrm{HCC}$ and prolongs survival in patients with T2 HCC.

Copyright $\odot 2007$ S. Karger AG, Basel

\section{Introduction}

Hepatic resection for hepatocellular carcinoma (HCC) has become a safe operation with a low operative mortality, and is the most effective treatment available at present [1-3]. However, the rates of intrahepatic recurrence of HCC are particularly high, even when curative hepatic resection can be performed [2]. Anatomic hepatectomy has been reported to be an effective treatment modality with a higher rate of survival or disease-free survival than that of limited hepatectomy, because it may prevent intrahepatic metastasis of HCC through the portal vein [4-8]. However, surgical outcomes between sectionectomy and segmentectomy have not been clarified in detail.

Vauthey et al. [9] reported a simplified staging system which is a modification of The American Joint Committee on Cancer (AJCC)/International Union Against Can-

\section{KARGER}

Fax +4161306 1234

E-Mail karger@karger.ch

www.karger.com (c) $2007 \mathrm{~S}$. Karger AG, Basel

$0253-4886 / 07 / 0245-0342 \$ 23.50 / 0$

Accessible online at:

www.karger.com/dsu
Shun-Ichi Ariizumi

Department of Surgery, Institute of Gastroenterology

Tokyo Women's Medical University

Kawada 8-1, Shinjuku-ku, Tokyo 162-0054 (Japan)

Tel. +81 33353 8111, Fax +8135269 7507, E-Mail ari@gd6.so-net.ne.jp 
Table 1. Surgical procedures for patients with T1 HCC and T2 HCC

\begin{tabular}{llr}
\hline T class & Surgical procedure & Patients \\
\hline T1 & Trisectionectomy & 5 \\
& Extended hemihepatectomy & 6 \\
& Hemihepatectomy & 45 \\
& Trisegmentectomy 4, 5,8 & 7 \\
Sectionectomy & 106 \\
Bisegmentectomy 5,6 or 7,8 or 4,5 or 4,8 & 50 \\
Segmentectomy & 105 \\
Partial segmentectomy & 82 \\
\hline T2 & 3 \\
& Trisectionectomy & 4 \\
& Hextended hemihepatectomy & 21 \\
Trisegmentectomy 4, 5, 8 & 5 \\
Sectionectomy & 39 \\
Bisegmentectomy 5,6 or 7,8 or 4,5 or 4,8 & 11 \\
Segmentectomy & 25 \\
Partial segmentectomy & 16 \\
\hline
\end{tabular}

cer (UICC) staging system for HCC. Recently, the UICC staging classification for HCC was modified on the basis of their report [10]. The effectiveness of anatomic hepatectomy according to the stage of HCC has not been clarified in detail. We attempted to clarify whether there might be a difference in outcome after sectionectomy or larger resection and segmentectomy or smaller resection in relation to the modified UICC T1 HCC and T2 HCC.

\section{Patients and Methods}

Between 1994 and 2003, 889 patients with HCC underwent initial hepatic resection for HCC at our institute. T1 HCC was diagnosed in 406, T2 HCC in 133, T3 HCC in 130, and T4 HCC in 17 by histopathological examination based on the UICC T classification. Nine of 133 patients with T2 HCC who underwent noncurative resection were excluded in this study. Patients with T3 HCC which invaded major portal or hepatic veins and patients with T4 HCC with distant metastasis or ruptured HCC were excluded in this study, because most patients with T3 HCC required hemihepatectomy or larger resection and in patients with T4 HCC curative resection could not be achieved with any hepatectomy. The remaining 203 patients who had been given a diagnosis of synchronous multicentric HCC by histopathological examination were excluded because if each of the multiple lesions includes a component of well-differentiated HCC, it is considered to indicate multicentric carcinogenesis and to be a primary cancer according to the General Rules for the Clinical and Pathological Study of Primary Liver Cancer of the Liver Cancer Study Group of Japan [11]. Surgical procedures for patients with T1 HCC and
T2 HCC are shown in table 1. The terminology of liver resection was determined based on the Terminology Committee of the International Hepato-Pancreato-Biliary Association in 2000 [12]. Patients who underwent left medial sectionectomy (segmentectomy 4) were included in the sectionectomy or larger resection group. Patients who underwent bisegmentectomy were included in the sectionectomy or larger resection group. We retrospectively examined surgical outcomes between sectionectomy or larger resection and segmentectomy or smaller resection in relation to the modified UICC T1 HCC and T2 HCC.

Sex, age, hepatitis $C$ virus antibody, serum level of $\alpha$-fetoprotein (AFP), Child-Pugh class, indocyanine green retention rate at 15 min $\left(\mathrm{ICGR}_{15}\right)$, tumor size, macroscopic type, histological grading, cirrhosis, and surgical procedure were examined. All surgical procedures were systematized hepatectomy with the Glissonean pedicle transection method $[7,13,14]$. The choice of resection was made on the basis of the tumor size, tumor type, and liver function $\left(\mathrm{ICGR}_{15}\right)$. In most patients with small and simple nodular HCC without daughter lesions, segmentectomy or smaller resection was performed. However, in patients with large and simple nodular HCC, sectionectomy or larger resection was performed considering functional liver reserve. In patients with HCC with daughter lesions or vascular invasion within the section, sectionectomy or larger resection was performed. However, in patients with HCC with daughter lesions or vascular invasion and poor functional liver reserve, segmentectomy or smaller resection was performed. Pathological findings including the macroscopic type were evaluated according to the General Rules for the Clinical and Pathological Study of Primary Liver Cancer of the Liver Cancer Study Group of Japan [11].

\section{Follow-Up}

After surgery, patients were followed up every 4-12 weeks at the outpatient department of our institution. Ultrasonography or computed tomography was performed once every 3-4 months. Intrahepatic recurrence was defined clinically as the appearance of a new lesion with radiological features typical of HCC, as confirmed by the above two imaging methods or biopsy specimens. Survival duration was defined as the time from hepatic resection to the date of death or last contact.

\section{Statistical Analysis}

Because of sample size limitations, patients with small nodular type with indistinct margins were combined with those with simple nodular type. Patients with single nodular with extranodular growth type, confluent multinodular type, massive type, and diffuse type were combined into a single group. Patients with moderately or poorly differentiated HCC, and undifferentiated HCC were also grouped together. Patients with Child-Pugh class $\mathrm{C}$ $(\mathrm{n}=1)$ were combined with those with Child-Pugh class B. The cumulative survival and recurrence-free survival rates among the patients were calculated by the Kaplan-Meier method and compared with the log-rank test. Univariate prognostic factors within each T1 class and T2 class were entered into a Cox's proportional hazard model to identify independent predictors of survival or recurrence. $p$ values $<0.05$ were taken to indicate statistical significance. We used StatView (Version 4.5 Hulinks, Tokyo, Japan) for statistical analysis. 
Table 2. Patient characteristics in relation to the surgical procedure in patients with T1 HCC

\begin{tabular}{lllr}
\hline & $\begin{array}{l}\text { Sectionectomy } \\
\text { or larger } \\
(\mathrm{n}=219)\end{array}$ & $\begin{array}{l}\text { Segmentectomy } \\
\text { or smaller } \\
(\mathrm{n}=187)\end{array}$ & p value \\
\hline Sex, male & 176 & 131 & 0.0159 \\
HCV antibody (positive) & 130 & 138 & 0.0022 \\
Cirrhosis (present) & 74 & 105 & $<0.0001$ \\
ICGR $_{15}(<15 \%)$ & 129 & 75 & 0.0001 \\
Child-Pugh class $(\mathrm{A})$ & 204 & 163 & 0.0414 \\
AFP $(<100$ ng/ml) & 173 & 146 & 0.7621 \\
Tumor size $(<3 \mathrm{~cm})$ & 114 & 137 & $<0.0001$ \\
Macroscopic type (simple nodular) & 154 & 159 & 0.0004 \\
Histological grade (moderately-poorly) & 198 & 136 & $<0.0001$ \\
\hline
\end{tabular}

$\mathrm{HCV}=$ Hepatitis $\mathrm{C}$ virus; $\mathrm{ICGR}_{15}=$ indocyanine green retention rate at $15 \mathrm{~min}$; $\mathrm{AFP}=\alpha$-fetoprotein.
Table 3. Patient characteristics in relation to the surgical procedure in patients with T2 HCC

\begin{tabular}{llll}
\hline & $\begin{array}{l}\text { Sectionectomy } \\
\text { or larger } \\
(\mathrm{n}=83)\end{array}$ & $\begin{array}{l}\text { Segmentectomy } \\
\text { or smaller } \\
\mathrm{n}=41)\end{array}$ & p value \\
\hline Sex, male & 68 & 28 & 0.0876 \\
HCV antibody (positive) & 52 & 28 & 0.5367 \\
Cirrhosis (present) & 23 & 26 & 0.0001 \\
ICGR $_{15},<15 \%$ & 50 & 14 & 0.0062 \\
Child-Pugh class (A) & 75 & 32 & 0.0607 \\
AFP (<100 ng/ml) & 39 & 25 & 0.2022 \\
Tumor size (<3 cm) & 25 & 26 & 0.0004 \\
Macroscopic type (simple nodular) & 27 & 16 & 0.4747 \\
Histological grade (moderately-poorly) & 82 & 41 & 0.4804 \\
Tumor number (multiple) & 36 & 19 & 0.7543 \\
Vascular invasion (present) & 64 & 32 & 0.9062 \\
\hline
\end{tabular}

$\mathrm{HCV}=$ Hepatitis $\mathrm{C}$ virus; $\mathrm{ICGR}_{15}=$ indocyanine green retention rate at $15 \mathrm{~min}$; $\mathrm{AFP}=\alpha$-fetoprotein.

\section{Results}

Patient characteristics in relation to the surgical procedure in patients with T1 and T2 HCC are shown in tables 2 and 3 . The number of patients with ICG $R_{15}$ $<15 \%$ was significantly lower in patients with $\mathrm{T} 1$ and $\mathrm{T} 2$ HCC who underwent segmentectomy or smaller resection than in patients with T1 and T2 HCC who underwent sectionectomy or larger resection. The number of cases in which tumor size $<3 \mathrm{~cm}$ was significantly higher in patients with T1 and T2 HCC who underwent segmentectomy or smaller resection than in patients with T1 and T2 HCC who underwent sectionectomy or larger resection.
Four of 530 patients died within 30 days after surgery. The overall recurrence-free survival curves and survival curves in relation to the surgical procedure in patients with $\mathrm{T} 1$ and $\mathrm{T} 2 \mathrm{HCC}$ are shown in figures 1 and 2. In patients with T1 HCC, the overall recurrence-free survival rate and survival rate were 45 and $79 \%$ in sectionectomy or larger resection, and 38 and $81 \%$ in segmentectomy or smaller resection, at 5 years, respectively. There were no significant differences between surgical procedures in patients with T1 HCC. However, in patients with T2 HCC, the 5-year recurrence-free survival rate and survival rate after sectionectomy or larger resection (37 and $71 \%$, respectively) were significantly different from those after segmentectomy (6\%: $\mathrm{p}<0.0001$, and $35 \%: \mathrm{p}=0.0027$, respectively). 
Fig. 1. Overall recurrence-free survival curves (a) and survival curves (b) of patients with UICC T1 HCC in relation to the surgical procedures are shown.

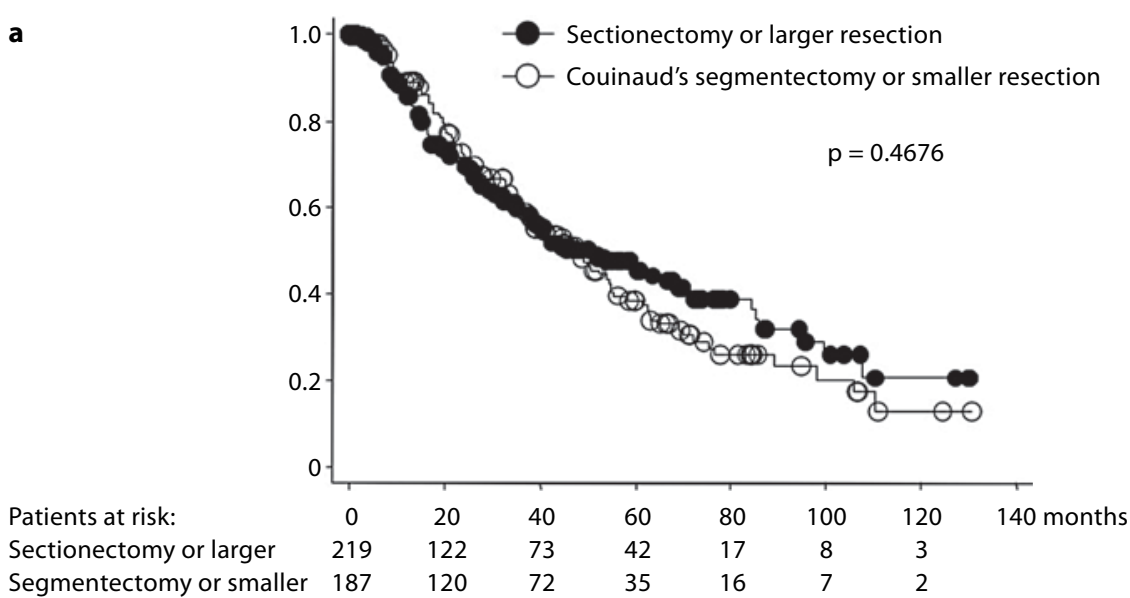

b

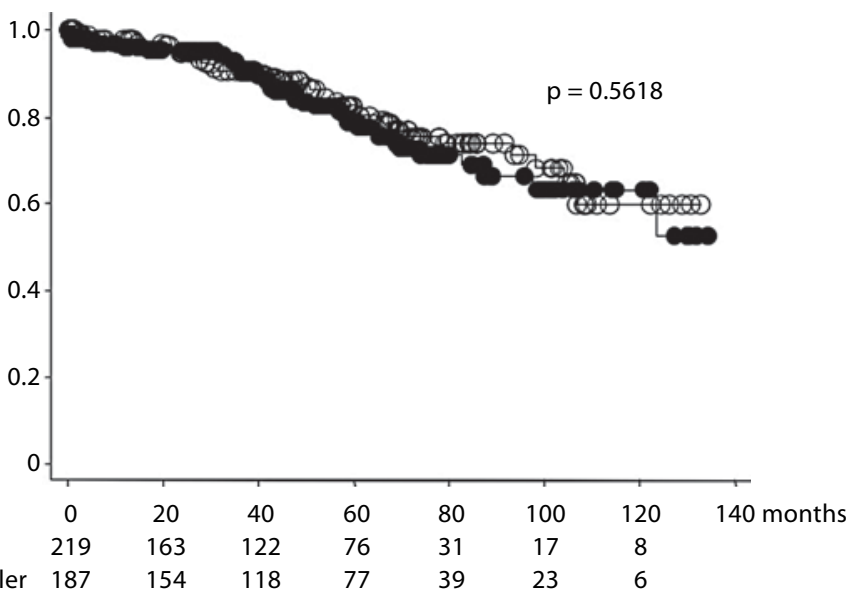

Patients at risk: Sectionectomy or larger Segmentectomy or smaller 187
Outcomes between surgical procedures were compared considering the liver function in patients with T2 HCC. In patients with T2 HCC with good liver function (ICGR15 $<15 \%$ ), the 5 -year recurrence-free survival rate after sectionectomy or larger resection (40\%) was significantly different from that after segmentectomy (13\%: $\mathrm{p}=0.0341)$. Even in patients with T2 HCC with ICGR15 $>15 \%$, the 5-year recurrence-free survival rate after sectionectomy or larger resection (30\%) was significantly different from that after segmentectomy $(0 \%$ : $\mathrm{p}=$ 0.0020).

The univariate analysis of prognostic factors of patients with T1 and T2 HCC is summarized in table 4. Univariate analysis showed that positive HCV antibody was significantly associated with recurrence-free survival in patients with T1 and T2 HCC. ICGR ${ }_{15}$ was significantly associated with recurrence-free survival and survival in patients with T1 and T2 HCC by univariate analysis.

The univariate prognostic factors were entered into a multivariate model to identify independent predictors of recurrence-free survival and survival. The factors associated with recurrence and survival by Cox's proportional hazard model are shown in table 5. Multivariate analysis showed the surgical procedure (sectionectomy or larger resection) to be a significant independent prognostic factor for recurrence-free survival and survival in patients with T2 HCC. 
Fig. 2. Overall recurrence-free survival curves (a) and survival curves (b) of patients with UICC T2 HCC in relation to the surgical procedures are shown. a

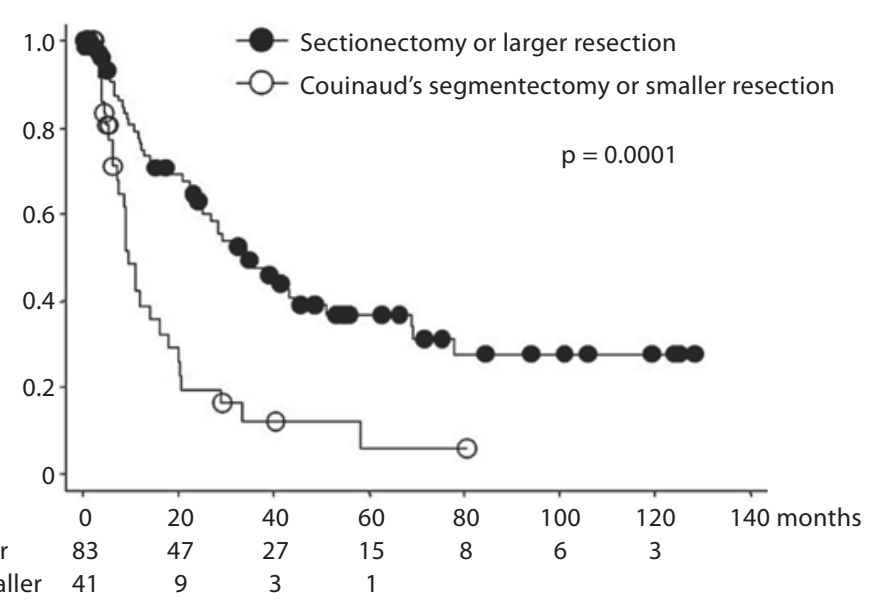

Sectionectomy or larger Segmentectomy or smaller

b

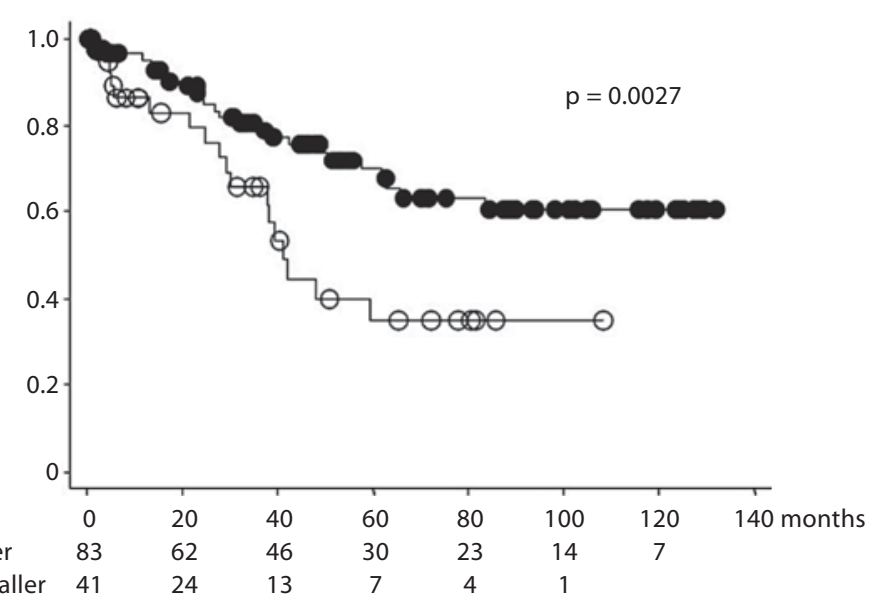

Patients at risk: Sectionectomy or larger Segmentectomy or smaller

\section{Discussion}

It is well known that vascular invasion of HCC, in particular portal vein invasion, is the best known HCC characteristic and prognostic factor in patients with HCC [2, 9, 15-17]. Because HCC often spreads around the section or whole liver through the portal vein, it is an important treatment strategy to target the section involving the tumor, in particular in patients with HCC with vascular invasion. According to the modified UICC classification [10], solitary HCC with vascular invasion is classified as $\mathrm{T} 2$, and HCC with invasion of a major branch of the portal or hepatic vein is classified as T3. If the HCC invades the portal vein, tumor cells such as of small intrahepatic metastasis caused by portal invasion cannot be removed completely, even by Couinaud's segmentectomy. Sectionectomy or larger resection is one of the most suitable modalities for treating the section including the tumor. In the present study, sectionectomy or larger resection was significantly associated with recurrence-free survival and survival by univariate analysis in patients with T2 HCC. Multivariate analysis also showed sectionectomy or larger resection to be a significant independent prognostic factor for recurrence-free survival and survival in patients with T2 HCC. Therefore, sectionectomy is the most suitable treatment modality for patients with T2 HCC, which has a higher risk of intrahepatic recurrence caused by intrahepatic metastasis. 
Table 4. Factors associated with recurrence-free survival rate and survival rate of patients by univariate analysis

\begin{tabular}{|c|c|c|c|c|c|}
\hline \multirow[t]{4}{*}{$\mathrm{T} 1$} & HCV antibody (negative vs. positive) & $60 / 33$ & $<0.0001$ & $84 / 78$ & 0.1682 \\
\hline & Cirrhosis (absent vs. present) & $48 / 33$ & 0.0060 & $82 / 77$ & 0.0097 \\
\hline & $\operatorname{ICGR}_{15}(<15$ vs. $>15 \%)$ & $52 / 29$ & 0.0004 & $88 / 71$ & 0.0003 \\
\hline & Child-Pugh class (A vs. B) & $43 / 35$ & 0.1647 & $83 / 40$ & $<0.0001$ \\
\hline \multirow[t]{5}{*}{$\mathrm{T} 2$} & Sex (male vs. female) & $31 / 15$ & 0.0240 & $63 / 49$ & 0.1570 \\
\hline & HCV antibody (negative vs. positive) & $41 / 19$ & 0.0110 & $69 / 56$ & 0.2569 \\
\hline & Cirrhosis (absent vs. present) & $31 / 22$ & 0.0245 & $71 / 44$ & 0.0586 \\
\hline & ICGR $_{15}(<15$ vs. $>15 \%)$ & $34 / 19$ & 0.0038 & $70 / 47$ & 0.0137 \\
\hline & Surgical procedure (sectionectomy vs. segmentectomy) & $37 / 6$ & $<0.0001$ & $71 / 35$ & 0.0027 \\
\hline
\end{tabular}

$\mathrm{HCV}=$ Hepatitis $\mathrm{C}$ virus; $\mathrm{ICGR}_{15}=$ indocyanine green retention rate at $15 \mathrm{~min}$; $\mathrm{AFP}=\alpha$-fetoprotein.

Table 5. Multivariate analysis using Cox's proportional hazards model

\begin{tabular}{|c|c|c|c|c|}
\hline & Risk factor & $\begin{array}{l}\text { Relative } \\
\text { risk }\end{array}$ & $95 \%$ CI & $\mathrm{p}$ value \\
\hline \multicolumn{5}{|c|}{ Recurrence } \\
\hline \multirow[t]{3}{*}{$\mathrm{T} 1$} & HCV antibody (positive) & 1.709 & $1.222,2.390$ & 0.0017 \\
\hline & $\operatorname{ICGR}_{15}(<15 \%)$ & 0.711 & $0.528,0.958$ & 0.0248 \\
\hline & Surgical procedure (sectionectomy or larger) & 1.121 & $0.843,1.491$ & 0.4330 \\
\hline \multirow[t]{3}{*}{$\mathrm{T} 2$} & HCV antibody (positive) & 2.071 & $1.228,3.493$ & 0.0063 \\
\hline & $\operatorname{ICGR}_{15}(<15 \%)$ & 0.571 & $0.351,0.929$ & 0.0239 \\
\hline & Surgical procedure (sectionectomy or larger) & 0.403 & $0.238,0.683$ & 0.0007 \\
\hline \multicolumn{5}{|c|}{ Survival } \\
\hline \multirow[t]{4}{*}{$\mathrm{T} 1$} & $\operatorname{ICGR}_{15}(<15 \%)$ & 0.485 & $0.291,0.811$ & 0.0058 \\
\hline & Child-Pugh class (A) & 0.432 & $0.230,0.814$ & 0.0094 \\
\hline & $\operatorname{AFP}(<100 \mathrm{ng} / \mathrm{ml})$ & 0.456 & $0.281,0.740$ & 0.0015 \\
\hline & Surgical procedure (sectionectomy or larger) & 1.574 & $0.971,2.549$ & 0.0654 \\
\hline \multirow[t]{2}{*}{$\mathrm{T} 2$} & $\operatorname{ICGR}_{15}(<15 \%)$ & 0.517 & $0.267,0.998$ & 0.0493 \\
\hline & Surgical procedure (sectionectomy or larger) & 0.437 & $0.228,0.837$ & 0.0125 \\
\hline
\end{tabular}

$\mathrm{CI}=$ Confidence interval; $\mathrm{HCV}=$ hepatitis $\mathrm{C}$ virus; $\mathrm{ICGR}_{15}=$ indocyanine green retention rate at $15 \mathrm{~min} ; \mathrm{AFP}=\alpha$-fetoprotein.
Originally the staging system for cancer requires not only an accurate prognostic assessment but also the true extent of tumor cell spread and a different treatment strategy within each stage. In Japan, the staging system of the General Rules for the Clinical and Pathological Study of Primary Liver Cancer of the Liver Cancer Study Group of Japan has been used [11]. This staging system has not been adopted worldwide, due to the complicated scoring system based on tumor size, number of tumors, and vascular or bile duct invasion, and it does not provide any guide on initial therapy for patients with HCC. The Barcelona Clinic Liver Cancer (BCLC) staging system provides an accurate prognostic assessment and only links the stage of the disease to a specific treatment strategy [18]. However, it does not refer to the surgical procedure such as sectionectomy or segmentectomy. The modified UICC staging system presents a true staging system considering tumor cell spread as vascular invasion, but it does not refer to the surgical procedure. However, the modified UICC staging system can link the stage to a 
specific surgical strategy in patients with T2 HCC because sectionectomy or larger resection for patients with T2 HCC was significantly associated with recurrencefree survival and survival in our present study.

It is also well known that liver function is an important prognostic factor for the recurrence-free survival and survival of patients with HCC $[1,2,4,9,17,19-21]$. In our present study, ICGR 15 was shown to be a significant independent prognostic factor for recurrence-free survival and survival in patients with T1 HCC on univariate and multivariate analysis. There was no difference in anatomic hepatectomy between sectionectomy or larger resection and segmentectomy or smaller resection for recurrence-free survival and survival by univariate and multivariate analysis. Since the outcome in patients with T1 HCC depends largely on liver function, anatomic resection which allows complete elimination of the HCC should be chosen considering liver function.
Anatomic hepatectomy resulting in excellent survival and recurrence-free survival of patients with solitary HCC has been reported. However, there are various procedures in anatomic hepatectomy. Sectionectomy and segmentectomy differ with regard to the extent of resection. In our present study, sectionectomy or larger resection for patients with T2 HCC was an independent significant factor for recurrence-free survival and survival. Therefore, sectionectomy prevents intrahepatic recurrence and prolongs survival in patients with T2 HCC.

\section{Acknowledgement}

The authors are indebted to Associate Professor Raoul Breugelmans of the International Medical Communications Center of Tokyo Medical University for his review of the manuscript.

\section{References}

1 Arii S, Yamaoka Y, Futagawa S, et al: Result of surgical and nonsurgical treatment for small-sized hepatocellular carcinomas: a retrospective and nationwide survey in Japan. Hepatology 2000;32:1224-1229.

-2 Iwao I, Arii S, Kojiro M, et al: Reevaluation of prognostic factors for survival after liver resection in patients with hepatocellular carcinoma in a Japanese nationwide survey. Cancer 2004;101:796-802.

3 Liver Cancer Study Group of Japan: Survey and follow-up study of primary liver cancer in Japan. Report 15. Acta Hepatol Jpn 2003; 44:157-175.

4 Kosuge T, Makuuchi M, Takayama T, et al: Long-term results after resection of hepatocellular carcinoma: experience of 480 cases. Hepatogastroenterology 1993;40:328-332.

5 Fuster J, Garcia-Valdecasas JC, Grande L, et al: Hepatocellular carcinoma and cirrhosis: results of surgical treatment in a European series. Ann Surg 1996;223:297-302.

-6 Regimbeau JM, Kianmanesh R, Farges O, et al: Extent of liver resection influences the outcome in patients with cirrhosis and small hepatocellular carcinoma. Surgery 2002;131: 311-317.
7 Yamamoto M, Takasaki K, Otsubo T, et al: Effectiveness of systematized hepatectomy with Glisson's pedicle transection at hepatic hilus for small nodular hepatocellular carcinoma: retrospective analysis. Surgery 2001; 130:443-448.

8 Hasegawa K, Kokubo N, Imamura H, et al: Prognostic impact of anatomic resection for hepatocellular carcinoma. Ann Surg 2005; 242:252-259

$\checkmark 9$ Vauthey JN, Lauwers GY, Esnaola NF, et al: Simplified staging for hepatocellular carcinoma. J Clin Oncol 2002;20:1527-1536.

10 International Union Against Cancer (UICC): TNM Classification of Malignant Tumours, ed 6. New York, Wiley-Liss, 2002, pp 81-83.

11 Liver Cancer Study Group of Japan: The General Rules for the Clinical and Pathological Study of Primary Liver Cancer, ed 2 Engl ed. Tokyo, Kanehara, 2003.

12 Terminology Committee of the International Hepato-Pancreato-Biliary Association: Modified from the Brisbane 2000 Terminology of liver anatomy and resections. HPB 2000;2:333-339.

13 Takasaki K, Kobayashi S, Tanaka S, et al: Highly anatomically systematized hepatic resection with Glissonean sheath cord transection at the hepatic hilus. Int Surg 1990;75: 73-77.

14 Takasaki K: Glissonean pedicle transection method for hepatic resection: a new concept of liver segmentation. J Hepatobiliary Pancreat Surg 1998;5:286-291.
15 Izumi R, Shimizu K, Ii T, et al: Prognostic factors of hepatocellular carcinoma in patients undergoing hepatic resection. Gastroenterology 1994;106:720-727.

16 Vauthey JN, Klimstra D, Franceschi D, et al: Factors affecting long-term prognosis after hepatic resection for hepatocellular carcinoma. Am J Surg 1995;169:28-35.

17 Tsai TJ, Chau GY, Lui WY, et al: Clinical significance of microscopic tumor venous invasion in patients with resectable hepatocellular carcinoma. Surgery 2000;127:603-608.

18 Llovet JM, Bru C, Bruix J: Prognosis of hepatocellular carcinoma: The BCLC staging classification. Semin Liver Dis 1999;19:329337.

19 Nagasue N, Kohno H, Chang YC, et al: Liver resection for hepatocellular carcinoma: results of 229 consecutive patients during 11 years. Ann Surg 1993;217:375-384.

20 Yamamoto J, Kosuge T, Takayama T, et al: Recurrence of hepatocellular carcinoma after surgery. Br J Surg 1996;83:1219-1222.

21 Bilimoria MM, Lauwers GY, Doherty DA, et al: Underlying liver disease, not tumor factors, predicts long-term survival after resection of hepatocellular carcinoma. Arch Surg 2001;136:528-535. 